\title{
ON THE SPECTRUM OF A NEW CLASS OF GRAPHS $^{1 *}$
}

\author{
Renny P VARGHESE ${ }^{1}$ \\ ${ }^{1}$ Department of Mathematics, Catholicate College, Pathanamthitta, Kerala, India, \\ rennypv1@gmail.com; ORCID:0000-0003-0243-979X
}

\section{ABSTRACT}

The pineapple graph $K_{n}^{t}$ is a graph of the form $K_{n}$ (complete graph of order $n$ ) with ' $t$ ' pendant edge attached to any one vertex of $K_{n}$. In this paper, we determined the adjacency Laplacian and signless Laplacian spectrum of pineapple graphs. Here we investigate some graph invariants like the number of spanning trees, Kirchhoff index and Laplacian-energy-like invariants of these graphs.

Keywords: Pineapple graph, Spectrum, Spanning tree, Kirchhoff index, Laplacian-energy-like invariant.

AMS Subject Classification (2010) : 05C50

\section{INTRODUCTION}

All graphs explained in this paper are undirected, without parallel edges and loops. Let $G=G(V, E)$ be a graph with vertex set $V(G)=\left\{v_{1}, v_{2}, \ldots, v_{n}\right\}$ and edge set $E(G)$. The adjacency matrix, $A(G)=\left(a_{i j}\right)_{n \times n}$, is an $n \times n$ symmetric matrix with rows and columns are indexed by vertices of $G$ where $a_{i j}=1$ if the vertices $v_{i}$ and $v_{j}$ are adjacent in $G, 0$ elsewhere. The characteristic polynomial of $A$ is of the form $f_{G}(A: x)=\operatorname{det}\left(x I_{n}-A\right)$ where $I_{n}$ is the identity matrix of order $n$. The roots of $f_{G}(A: x)=0$ constitute the eigenvalues of $G$.

Let $d_{i}$ be the diagonal degree of the vertex $v_{i}$ in $G$ and $D(G)=\operatorname{diag}\left(d_{1}, d_{2}, \cdots d_{n}\right)$ be the diagonal matrix of $G$. The Laplacian matrix and signless Laplacian matrix of the graph $G$ are defined as $L(G)=D(G)-A(G)$ and $Q(G)=D(G)+A(G)$ respectively. The eigenvalues of $A, L$ and $Q$ are respectively called the $A$-spectrum, $L$-spectrum and $Q$-spectrum of the graph $G$. Some new graphs are defined and their spectrum are calculated in $[3,6,7,8]$.

In this paper, we are interested in finding the Laplacian and signless Laplacian spectrum

1* Received: 20.07.2021 - Accepted: 11.08.2021

DOI: 10.17932/EJEAS.2021.024/ejeas_v01i2005 
of the pineapple graphs. Also, as an application,we investigated the Kirchhoff index, the number of spanning trees and Laplacian-energy-like invariants of the pineapple graphs.

The arrangement of the paper in section wise as follows. Section 2, describes the necessary preliminaries. In section 3, we determine the Laplacian and signless Laplacian spectrum of pineapple graphs and some applications such as the number of spanning trees, Kirchhoff index and Laplacian-energy-like invariants of the graphs.

\section{PRELIMINARIES}

Definition 2.1 A pineapple graph is a graph of the form $K_{n}$ with $t$ pendant edge attached to any one vertices of the complete graph $K_{n}$. We denote this graph by $K_{n}^{t}$.

As we know $K_{n}^{t}$ has $(n+t)$ vertices and $\left(\begin{array}{l}n \\ 2\end{array}\right)+t$ edges. Also, we assume that $n \geq 3$ and $t \geq 1$.

Theorem 2.2 [5, 9] The spectrum of the pineapple graph $K_{n}^{t}$ with $(n+t)$ vertices consists of,

(i) $\quad-1, \quad$ repeated $(n-2)$ times

(ii) $\quad 0$, repeated $(t-1)$ times

(iii) Three roots of the cubic $x^{3}-(n-2) x^{2}-(n+t-1) x-(n-2) t=$ 0 .

Proof. Let $G=K_{n}^{t}$ be a pineapple graphs on $(n+t)$ vertices. The adjacency matrix of $G$ is,

$$
A=\left[\begin{array}{lll}
0 & J_{1 \times(n-1)}^{T} & J_{1 \times t}^{T} \\
J_{(n-1) \times 1} & J_{1 \times(n-1)}-I_{n} & 0_{n \times t} \\
J_{t \times 1} & 0_{t \times(n-1)} & I_{t}
\end{array}\right],
$$

where $J$ is the all one matrix of appropriate order and $I_{n}$ is the identity matrix of order $n$.

It can be proved that the characteristic polynomial of $G$ is,

$f_{G}(A: x)=x^{t-1}(x+1)^{p-2}\left(x^{3}-x^{2}(n-2)-(n+t-1) x+t(n-2)\right)$.

Example : The following graph represents a particular example of pineapple graph. 


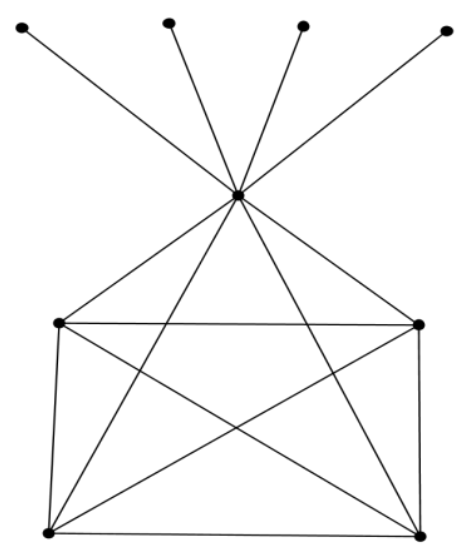

Figure 1: Pineapple graph $K_{5}^{4}$

\section{LAPLACIAN SPECTRUM OF PINEAPPLE GRAPH}

In this section, we determine the Laplacian and signless Laplacian spectrum of pineapple graphs.

Theorem 3.1 Let $K_{n}^{t}$ be a pineapple graph with $(n+t)$ vertices. Then $L$-spectrum of $K_{n}^{t}$ consists of,
(i) 0 ;
(ii) $n+p$;
(iii) 1 , repeated $t$ times;
(iv) $n$, repeated $(n-2)$ times.

Proof. Let $t$ pendant vertices $x_{j}, j=1,2, \ldots, t$ is attached to the vertex $v_{1}$ of the pineapple graph. The degree of the vertices of $K_{n}^{t}$ are $d\left(v_{1}\right)=n-1+t$, $d\left(v_{i}\right)=n-1, i=2, \ldots, n$ and $d\left(x_{j}\right)=1, j=1,2, \ldots, t$.

The diagonal degree matrix of the pineapple graph is,

$$
D\left(K_{n}^{t}\right)=\left[\begin{array}{lll}
n+t-1 & 0 & 0 \\
0 & (n-1) I_{n-1} & 0 \\
0 & 0 & I_{t}
\end{array}\right] .
$$

The Laplacian matrix of $K_{n}^{t}$ is,

$$
L=D-A
$$


On The Spectrum of a New Class of Graphs

$$
=\left[\begin{array}{lll}
n+t-1 & -J_{1 \times(n-1)}^{T} & -J_{1 \times t}^{T} \\
-J_{(n-1) \times 1} & n I_{n-1}-J_{n-1} & 0_{n \times t} \\
-J_{t \times 1} & 0_{t \times(n-1)} & I_{t}
\end{array}\right],
$$

Let $1_{n}$ denote the eigenvector corresponding to the eigenvalue $(n-1)$ of $G$, then A. $1_{n}=(n-1) 1_{n}$.

Let $\left(\alpha, \beta 1_{n-1}, \gamma 1_{t}\right)$ be a non zero vector.

$$
\begin{array}{r}
(n+t-1) \alpha-(n-1) \beta-t \gamma=x \alpha \\
-\alpha+\beta+0 . \gamma=x \beta \\
-\alpha+0 . \beta+\gamma=x \gamma
\end{array}
$$

From equation (3.2), we get $\beta=\frac{-\alpha}{x-1}$.

From equation (3.3), we arrive at $\gamma=\frac{-\alpha}{x-1}$.

Substitute these values in (3.1), we get

Since $\alpha \neq 0$ we arrive at,

$$
(n+t-1) \alpha+\frac{(n-1) \alpha}{x-1}+\frac{p \alpha}{x-1}=x \alpha .
$$

$$
\begin{aligned}
(n+t-1)(x-1)+n-1+t & =x(x-1) \\
n x-n+t x-t-x+1+n-1+t & =x(x-1) \\
n x+t x & =x^{2} \\
x^{2}-(n+t) x & =0 \\
x & =0, n+t .
\end{aligned}
$$

Consider $U=\left(\begin{array}{l}0 \\ 0 \\ X\end{array}\right)$, where $X$ is a $t \times 1$ vector.

$$
\begin{aligned}
L U & =\left[\begin{array}{lll}
n+t-1 & -J_{1 \times(n-1)}^{T} & -J_{1 \times t}^{T} \\
-J_{(n-1) \times 1} & n I_{n-1}-J_{n-1} & 0_{n \times t} \\
-J_{t \times 1} & 0_{t \times(n-1)} & I_{t}
\end{array}\right]\left(\begin{array}{l}
0 \\
0 \\
X
\end{array}\right) \\
& =\left(\begin{array}{l}
-J^{T} X \\
0 \\
I_{t} X
\end{array}\right) \\
& =\left(\begin{array}{l}
0 \\
0 \\
X
\end{array}\right)=U .
\end{aligned}
$$

Hence $\mu=1$ is an eigenvalue corresponding to $U$, which repeats $t$ times.

Consider $V=\left(\begin{array}{l}0 \\ Y \\ 0\end{array}\right)$, where $Y$ is a $(n-1) \times 1$ vector, 


$$
L V=\left(\begin{array}{l}
-J^{T} Y \\
(n I-J) Y \\
0
\end{array}\right)=\left(\begin{array}{l}
0 \\
n Y \\
0
\end{array}\right)=n V .
$$

Hence $\mu=n$ is an eigenvalue corresponding to $V$, which repeats $(n-1)$ times. Thus the theorem is proved.

\subsection{THE NUMBER OF SPANNING TREES}

Spanning tree of a graph is a subgraph of it which is also a tree. The number of spanning tree of a graph $G$ is denoted by $t(G)$. If $G$ is a connected graph with $n$ vertices and the Laplacian spectrum $0=\mu_{1}(G) \leq \mu_{2}(G) \cdots \leq \mu_{n}(G)$ then [1] the number of spanning trees,

$$
t(G)=\frac{\mu_{2}(G) \mu_{3}(G) \cdots \mu_{n}(G)}{n} .
$$

Theorem 3.2 Let $G=K_{n}^{p}$ be a pineapple graph with $(n+p)$ vertices. Then, $t(G)=$ $t\left(K_{n}\right)$.

Proof. Referring the notations used in Theorem 3.1.

The Laplacian spectrum consists of 1 repeated $p$ times and $n$ repeated $(n-2)$ times together with 0 and $(n+p)$.

Substituting these result in the equation (3.4) we get,

$$
\begin{aligned}
t(G) & =\frac{\mu_{2}(G) \mu_{3}(G) \cdots \mu_{n}(G)}{n} \\
& =\frac{1}{(n+p)}\left[(n+p) \cdot n^{n-2}\right] \\
& =n^{n-2} .
\end{aligned}
$$

We know that for a complete graph of order $n$, the number of spanning tree, $t\left(K_{n}\right)=$ $n^{n-2}$.

$$
\text { Hence } t(G)=t\left(K_{n}\right) \text {. }
$$

\subsection{KIRCHHOFF INDEX}

Randić and Klein in [2] introduced a new notion named resistance distance, based on electric resistance in a network corresponding to a graph. Here the resistance distance between any two adjacent vertices is chosen as $1 \mathrm{ohm}$. By means of the Kirchhoff laws, the electric resistance is calculated and is called kirchhoff index. For a graph $G$ with $n \quad(n \geq 2)$ vertices the Kirchhoff index, $K f(G)$, is defined as,

$$
K f(G)=n \sum_{i=2}^{n} \frac{1}{\mu_{i}} .
$$


Theorem 3.3 Let $G=K_{n}^{p}$ be a pineapple graph with $(n+p)$ vertices. Then,

$$
K f(G)=1+\frac{(n+p)(n p+n-2)}{n} .
$$

Proof. We know that for a pineapple graph, the Laplacian spectrum consists of $0, n+p$, 1 repeated $p$ times and $n$ repeated $(n-2)$ times by referring 3.1. Substituting these result in the equation (3.5) we get,

$$
\begin{aligned}
K f(G) & =n \sum_{i=2}^{n} \frac{1}{\mu_{i}} \\
& =(n+p)\left[\frac{1}{n+p}+p+\frac{n-2}{n}\right] \\
& =1+p(n+p)+\frac{(n+p)(n-2)}{n} \\
& =1+\frac{(n+p)(n p+n-2)}{n} .
\end{aligned}
$$

\subsection{LAPLACIAN-ENERGY-LIKE INVARIANT}

A new graph invariant based on Laplacian spectrum called, Laplacian-energy-like invariant was defined by B. Liu and Liu in [4]. The Laplacian-energy-like invariant (LEL) of a graph $G$ with $n$ vertices is defined as,

$$
\operatorname{LEL}(G)=\sum_{i=2}^{n} \sqrt{\mu_{i}} \text {. }
$$

Theorem 3.4 Let $G=K_{n}^{p}$ be a pineapple graph with $(n+p)$ vertices. Then Laplaceenergy-like invariant, $L E L(G)=p+(n-2) \sqrt{n}+\sqrt{n+p}$.

Proof. Using the Theorem 3.1,

$$
\begin{aligned}
\operatorname{LEL}(G)=\sum_{i=2}^{n} \sqrt{\mu_{i}} & \\
=\sqrt{n+p}+p+(n-2) \sqrt{n} & \\
& =p+(n-2) \sqrt{n}+\sqrt{n+p} .
\end{aligned}
$$

\subsection{SIGNLESS LAPLACIAN SPECTRUM OF PINEAPPLE GRAPH}

Here we find the signless Laplacian spectrum (Q-spectrum) of pineapple graphs.

Theorem 3.5 Let $K_{n}^{t}$ be a pineapple graph with $n+t$ vertices. Then $Q$-spectrum of $K_{n}^{t}$ consists of,

(i) 1 , repeated $(t-1)$ times ;

(ii) $(n-2)$, repeated $(n-2)$ times; 
(iii) Three roots of the cubic,

$$
x^{3}-(3 n+p-3) x+(n+p)(2 n-3) x-2(n-1)(n-2)=0 \text {. }
$$

Proof. The degree of the vertices of $K_{n}^{t}$ are $d\left(v_{1}\right)=n-1+t, d\left(v_{i}\right)=n-1, i=$ $2, \ldots, n$ and $d\left(x_{j}\right)=1, j=1,2, \ldots, t$.

The diagonal degree matrix of the pineapple graph is, The signless Laplace matrix of $K_{n}^{t}$ is,

$$
\begin{aligned}
Q & =D-A \\
& =\left[\begin{array}{lll}
n+t+1 & J^{T} & J^{T} \\
J & (n-2) I+J_{n-1} & 0 \\
J & 0 & I_{t}
\end{array}\right],
\end{aligned}
$$

We have the $Q$-spectrum of the complete graph $K_{n}$ is $(n-2)$, repeated $(n-1)$ times and $(2 n-2)$, repeated 1 time.

$$
\text { Consider } \begin{aligned}
U & =\left(\begin{array}{l}
0 \\
0 \\
X
\end{array}\right) \text {, where } X \text { is a } t \times 1 \text { vector. } \\
Q U & =\left[\begin{array}{lll}
n+t+1 & J^{T} & J^{T} \\
J & (n-2) I+J_{n-1} & 0 \\
J & 0 & I_{t}
\end{array}\right]\left(\begin{array}{l}
0 \\
0 \\
X
\end{array}\right) \\
& =\left(\begin{array}{l}
J X \\
0 \\
I_{t} X
\end{array}\right) \\
& =\left(\begin{array}{l}
0 \\
0 \\
X
\end{array}\right)=U .
\end{aligned}
$$

Hence $v=1$ is an eigenvalue corresponding to $U$, which repeated $(t-1)$ times. Consider $V=\left(\begin{array}{l}0 \\ Y \\ 0\end{array}\right)$, where $Y$ is a $(n-1) \times 1$ vector,

$$
\begin{aligned}
& Q V=\left(\begin{array}{l}
J Y \\
((n-2) I+J) Y \\
0
\end{array}\right) \\
& \quad=\left(\begin{array}{l}
0 \\
(n-2) Y \\
0
\end{array}\right)=(n-2) V
\end{aligned}
$$


Hence $v=n-2$ is an eigenvalue corresponding to $V$ which repeated $(n-1)-1=$ $n-2$ times.

So there are $n-2+t-1=n+t-3$ eigenvectors of $Q$.

The remaining four eigenvectors of $Q$ are of the form $\left(\alpha, \beta 1_{n-1}, \gamma 1_{t}\right)$ for some $(\alpha, \beta, \gamma) \neq 0$.

Let $x$ be an eigenvalue of $Q$ corresponding to $\left(\alpha, \beta 1_{n-1}, \gamma 1_{t}\right)^{T}$ we get a system of equation,

$$
\begin{array}{r}
(n+t-1) \alpha-(n-1) \beta+t \gamma=x \alpha \\
\alpha+(n-2+n-1) \beta+0 \cdot \gamma=x \beta \\
\alpha+0 . \beta+\gamma=x \gamma
\end{array}
$$

From equation (3.8), we get $\alpha=x-2 n+3 \beta$, then we get $\beta=\frac{\alpha}{x-2 n+3}$.

From equation (3.9), we arrive at $\alpha=x-1 \gamma$, which implies $\gamma=\frac{\frac{x-2 n}{\alpha}}{x-1}$.

If $\alpha=0$ then $\beta=\gamma=0$. So assume $\alpha=0$.

Substitute the value of $\beta$ and $\gamma$ in equation (3.7) we get,

$$
\begin{gathered}
(n+t-1)+\frac{(n-1) \alpha}{x-2 n+3}+\frac{t \alpha}{x-1}=x \alpha . \\
(n+t-1)(x-2 n+3)(x-1)+(n-1) 9 x-1)+t(x-2 n+3) \\
=x(x-1)(x-2 n+3) .
\end{gathered}
$$

On simplifying we get,

$x^{3}-(3 n+t-3) x^{2}+\left(2 n^{2}+2 n t-3 n-3 t\right) x-2\left(n^{2}-3 n+2\right)=0$.

From these arguments, all the spectral values are calculated.

\section{CONCLUSION}

Determining and analysing the spectrum of graphs is an important and exciting research topic in Mathematics and in theoretical Computer Science. Several graph spectra are introduced by researchers to get deep information about a graph. The main idea of spectral graph theory is to exploit the relation between graphs and matrices. This is possible with the help of eigenvalues and eigenvectors of some graph matrices. In addition to this, we determined such spectrum of pineapple graphs. Also, we investigate some graph invariants like the number of spanning trees, Kirchhoff index and Laplacian-energy-like invariants of such graphs.

\section{REFERENCES}

[1] D. M. Cvetkovic', M. Doob, H. Sachs, Spectra of Graphs, Theory and Applications, Third edition, Johann Ambrosius Barth Heidelberg, (1995).

[2] D. J. Klein, M. Randic, "Resistance distance”, J. Math. Chem., 81 - 95, 1993. 
[3] K. R. Kumar, R. P. Varghese, "Spectrum of (k, r)-regular hypergraphs", Int. J. of Math. Combin., vol. 2, pp.52-59, 2017.

[4] J. Liu, B. Liu, “A Laplacian-energy-like invarient of a graph”, MATCH Commun. Math. comput. Chem., vol. 59, pp.397 - 413, 2008.

[5] H. Topcu, S. Sorgun, W. H. Haemers, "On the spectral characterization of pineapple Graphs",

Linear Algebra Appl., vol. 507, pp.267-273, 2016.

[6] R. P. Varghese, D. Susha, "The spectrum of two new corona of graphs and its Applications”, Int. J. Math. And its Appl., vol. 5(4), pp.395-406, 2017.

[7] R. P. Varghese, K. R. Kumar, "Spectra of a new join of two graphs, Advances in Theoretical and Applied Mathematics", vol. 11(4), pp. 459-470, 2016.

[8] R. P. Varghese, D. Susha, "On the normalized Laplacian spectrum of some graphs”, Kragujevac J. Math., vol. 44(3), pp.431-442, 2020.

[9] X. Zhang, H. Zhang, "Some graphs determined by their spectra", Linear Algebra Appl., vol. 431, pp.1443-1454, 2009. 